\title{
ON WEYL FRACTIONAL CALCULUS
}

\author{
R. K. RAINA AND C. L. KOUL
}

ABstract. The Weyl fractional calculus is applied in developing the Laplace transform of $t^{q} f(t)$, for all values of $q$. Also, a generalized Taylor's formula in Weyl fractional calculus is established. The results are then used in deriving a certain generating function for the $\boldsymbol{H}$-function of Fox.

1. Introduction. Let $\boldsymbol{A}$ denote a class of good functions. By a good function $f$, we mean (Miller [6, p. 82]) a function which is everywhere differentiable any number of times and if it and all of its derivatives are $O\left(x^{-v}\right)$, for all $v$ as $x$ increases without limit.

We define the Weyl fractional derivatives of a function $g(z)$ as follows: Let $g \in A$, then

$$
z^{D_{\infty}^{q} g(z)}=\frac{(-1)^{q}}{\Gamma(-q)} \int_{z}^{\infty}(u-z)^{-q-1} g(u) d u, \quad \text { for } q<0 .
$$

For $q \geq 0$,

$$
z^{D_{\infty}^{q} g(z)}=\frac{d^{r}}{d z^{r}}\left(z^{D_{\infty}^{q-r} g(z)}\right),
$$

$r$ being a positive integer such that $r>q$.

We recall the definition of $H$-function of Fox [3, p. 408] in the form:

$$
\begin{aligned}
H(z) & =H_{P, Q}^{M, N}\left[\begin{array}{c}
z \\
\left(a_{i}, \alpha_{i}\right)_{1, P} \\
\left(b_{i}, \beta_{i}\right)_{1, Q}
\end{array}\right] \\
& =\frac{1}{2 \pi \omega} \int_{L} \theta(s) z^{s} d s, \quad \omega=\sqrt{-1},
\end{aligned}
$$

where, for convenience,

$$
\theta(s)=\frac{\prod_{i=1}^{M} \Gamma\left(b_{i}-\beta_{i} s\right) \prod_{i=1}^{N} \Gamma\left(1-a_{i}+\alpha_{i} s\right)}{\prod_{i=M+1}^{Q} \Gamma\left(1-b_{i}+\beta_{i} s\right) \prod_{i=N+1}^{P} \Gamma\left(a_{i}-\alpha_{i} s\right)},
$$

$z \neq 0$, and an empty product is interpreted as unity. The integers $M, N, P, Q$, are such that $0<M<Q, 0<N \leqslant P$; the coefficients $\alpha_{i}(i=1, \ldots, P), \beta_{i}$

Received by the editors March 1, 1978.

AMS (MOS) subject classifications (1970). Primary 26A33, 44A10; Secondary 33A35.

Key words and phrases. Weyl fractional calculus, H-function, Laplace transform, generalized Taylor's formula, generating functions. 
$(i=1, \ldots, Q)$, are all assumed to be positive. $L$ is a suitably chosen contour such that all the poles of $\theta(s)$ are simple.

The $H$-function $H(z)$ is a very general function and has for its particular cases a number of important special functions. For the conditions of existence of the function (3) and its various special cases, the paper of Gupta and Jain [4, pp. 596-600] may be referred to.

\section{The Laplace transform of $t^{q} f(t)$, for arbitrary $q$.}

THEOREM 1. Let $f(t)$ be such that

$$
g(p)=L[f(t) ; p] \text { exists and belongs to } A,
$$

where $L[f(t) ; p]$ denotes the Laplace transform of $f(t)$. Then, for all $q$,

$$
(-1)^{q} p^{D_{\infty}^{q} g(p)}=L\left[t^{q} f(t) ; p\right]
$$

Proof. For $q<0$, we have in view of (1)

$$
\begin{aligned}
(-1)^{q} p^{D_{\infty}^{q} g(p)} & =\frac{1}{\Gamma(-q)} \int_{p}^{\infty}(u-p)^{-q-1} g(u) d u \\
& =\frac{1}{\Gamma(-q)} \int_{p}^{\infty}\left((u-p)^{-q-1} \int_{0}^{\infty} e^{-u t} f(t) d t\right) d u \\
& =\frac{1}{\Gamma(-q)} \int_{0}^{\infty} f(t)\left(\int_{p}^{\infty}(u-p)^{-q-1} e^{-u t} d u\right) d t
\end{aligned}
$$

Using Erdélyi [2, p. 202, (11)] to evaluate the inner integral on the R.H.S, we get

$$
(-1)^{q} p^{D_{\infty}^{q} g(p)}=L\left[t^{q} f(t) ; p\right], \text { for } q<0 .
$$

For $q>0$, invoking the definition (2), we can write:

$$
(-1)^{q} p^{D_{\infty}^{q} g(p)}=\frac{d^{r}}{d p^{r}}\left(\int_{0}^{\infty} e^{-p t} t^{q-r} f(t) d t\right)
$$

Differentiating under the sign of integral, we again find that

$$
(-1)^{q} p^{D_{\infty}^{q} g(p)}=L\left[t^{q} f(t) ; p\right], \text { for } q \geqslant 0 .
$$

This completes the proof of the theorem.

3. The generalized Taylor's formula. We prove the following theorem which may be regarded as a generalization of the familiar Taylor's formula.

THEOREM 2. Let

(i) $a$ be a real number such that $0<a \leqslant 1$,

(ii) $\eta$ be an arbitrary complex number and

(iii) $g(p) \in A$.

Then

$$
g(p+t)=\sum_{n=-\infty}^{\infty} \frac{a t^{a n+\eta}}{\Gamma(a n+\eta+1)} p^{D_{\infty}^{a n+\eta} g(p)}
$$

is valid for all $t$ on the circle $|t / p|=1$. 
Proof. Since $g \in A$, we may suppose $g(z)=z^{-\lambda} f(z)$. Assume $f(z)=$ $\sum_{r=0}^{\infty} C_{r} z^{r}$.

The L.H.S. of (10) then gives $(p+t)^{-\lambda} \sum_{r=0}^{\infty} C_{r}(p+t)^{r}$. Now the R.H.S. of $(10)$ is

$$
\begin{aligned}
\sum_{n=-\infty}^{\infty} \frac{a t^{a n+\eta}}{\Gamma(a n+\eta+1)} p^{D_{\infty}^{a+n}}\left(p^{-\lambda} \sum_{r=0}^{\infty} C_{r} p^{r}\right) \\
=\sum_{n=-\infty}^{\infty} \frac{a t^{a n+\eta}}{\Gamma(a n+\eta+1)} \sum_{r=0}^{\infty} C_{r} \cdot p^{D_{\infty}^{a n+\eta} p^{-(\lambda-r)}}
\end{aligned}
$$

Since

$$
z^{D_{\infty}^{q} z^{-\lambda}}=\frac{(-1)^{q} \Gamma(\lambda+q)}{\Gamma(\lambda)} z^{-\lambda-q},
$$

valid for all $q$, the R.H.S. of (10) after a little simplification reduces to

$$
\begin{gathered}
p^{-\lambda} \sum_{r=0}^{\infty} C_{r} \cdot p^{r} \sum_{n=-\infty}^{\infty} a\left(\begin{array}{c}
\lambda-r+a n+\eta-1 \\
a n+\eta
\end{array}\right)\left(-\frac{t}{p}\right)^{a n+\eta} \\
=p^{-\lambda} \sum_{r=0}^{\infty} C_{r} p^{r} \sum_{n=-\infty}^{\infty} a\left(\begin{array}{c}
-\lambda+r \\
a n+\eta
\end{array}\right)\left(\frac{t}{p}\right)^{a n+\eta}, \\
\text { since }(-1)^{r}\left(\begin{array}{c}
n \\
r
\end{array}\right)=\frac{(-n) r}{r !} .
\end{gathered}
$$

The inner sum can be simplified by making use of the known formula of Osler [7, p. 46, (5.1)], viz.

$$
\sum_{n=-\infty}^{\infty} a\left(\begin{array}{c}
p \\
\text { an }+\eta
\end{array}\right) t^{a n+\eta}=(1+t)^{p}, \text { for }|t|=1,
$$

to get $\sum_{r=0}^{\infty} C_{r}(p+t)^{-\lambda+r}$. This completes the proof of Theorem 2.

\section{Generating functions. Suppose}

$$
f(t)=t^{\sigma-1} H_{P, Q}^{M, N}\left[a t^{h}\left[\begin{array}{l}
\left(a_{i}, \alpha_{i}\right)_{1, P} \\
\left(b_{i}, \beta_{i}\right)_{1, Q}
\end{array}\right], \quad h>0 .\right.
$$

Then from (5) and Gupta [5, p. 83],

provided that

$$
g(p)=p^{-\sigma} H_{P+1, Q}^{M, N+1}\left[a p^{-h} \mid \begin{array}{c}
(1-\sigma, h),\left(a_{i}, \alpha_{i}\right)_{1, P} \\
\left(b_{i}, \beta_{i}\right)_{1, Q}
\end{array}\right],
$$

$$
\operatorname{Re}\left[\sigma+h\left(b_{i} / \beta_{i}\right)\right]>0 \quad(i=1, \ldots, M), \quad|\arg a|<\frac{1}{2} K \pi,
$$

where

$$
K=\sum_{1}^{M}\left(\beta_{i}\right)-\sum_{M+1}^{Q}\left(\beta_{i}\right)+\sum_{1}^{N}\left(\alpha_{i}\right)-\sum_{N+1}^{P}\left(\alpha_{i}\right)>0
$$


Also, we have for arbitrary $q$,

$$
\begin{aligned}
& z^{D_{\Phi_{Z}}^{q} Z^{\lambda} H_{P, Q}^{M, N}}\left[a z^{-h} \mid \begin{array}{c}
\left(a_{i}, \alpha_{i}\right)_{1, P} \\
\left(b_{i}, \beta_{i}\right)_{1, Q}
\end{array}\right] \\
& =(-1)^{q} z^{\lambda-q} H_{P+1, Q+1}^{M, N+1}\left[a z^{-h} \mid \begin{array}{c}
(1+\lambda-q, h),\left(a_{i}, \alpha_{i}\right)_{1, P} \\
\left(b_{i}, \beta_{i}\right)_{1, Q},(1+\lambda, h)
\end{array}\right],
\end{aligned}
$$

provided that

$$
\begin{aligned}
\operatorname{Re}\left[-\lambda+h_{\beta_{i}}^{b_{i}}\right]>0 & (i=1, \ldots, M), h>0,|\arg a|<\frac{1}{2} K \pi, \\
& \text { where } K=\sum_{1}^{M}\left(\beta_{i}\right)-\sum_{M+1}^{Q}\left(\beta_{i}\right)+\sum_{1}^{N}\left(\alpha_{i}\right)-\sum_{N+1}^{P}\left(\alpha_{i}\right)>0 .
\end{aligned}
$$

(14a) can be established easily by using the contour integral representation (3) and (11).

Substituting $f(t)$ and $g(p)$ from (13) and (14) respectively in the generalized Taylor's formula (10) and using (14a) appropriately, we get

$$
\begin{gathered}
(p+t)^{-o} H_{P+1, Q}^{M, N+1}\left[a^{\prime}(p+t)^{-h} \mid \begin{array}{c}
(1-\sigma, h),\left(a_{i}, \alpha_{i}\right)_{1, P} \\
\left(b_{i}, \beta_{i}\right)_{1, Q}
\end{array}\right] \\
=p^{-\sigma} \sum_{n=-\infty}^{\infty} \frac{a(-t / p)^{a n+\eta}}{\Gamma(a n+\eta+1)} \\
\cdot H_{P+1, Q}^{M, N+1}\left[a^{\prime} p^{-h} \mid \begin{array}{c}
(1-\sigma-a n-\eta, h),\left(a_{i}, \alpha_{i}\right)_{1, P} \\
\left(b_{i}, \beta_{i}\right)_{1, Q}
\end{array}\right] .
\end{gathered}
$$

Putting $p=1, \sigma=1-a_{1}, h=\alpha_{1}$ and adjusting the other parameters, we get the generating function

$$
\begin{aligned}
& (1+t)^{a_{1}-1} H_{P, Q}^{M, N}\left[z(1+t)^{-\alpha_{1} \mid} \begin{array}{c}
\left(a_{i}, \alpha_{i}\right)_{1, P} \\
\left(b_{i}, \beta_{i}\right)_{1, Q}
\end{array}\right] \\
& =\sum_{n=-\infty}^{\infty} \frac{a(-t)^{a n+\eta}}{\Gamma(a n+\eta+1)} H_{P, Q}^{M, N}\left[z \mid \begin{array}{c}
\left(a_{1}-a n-\eta, \alpha_{1}\right),\left(a_{i}, \alpha_{i}\right)_{2, P} \\
\left(b_{i}, \beta_{i}\right)_{1, Q}
\end{array}\right] .
\end{aligned}
$$

Obviously for $\eta=0$ and $a=1$, (16) corresponds to a known result of Anandani [1, p. 6, (2.1)].

\section{REFERENCES}

1. P. Anandani, On some generating functions for $\boldsymbol{H}$-functions, Labdev J. Sci. Tech. India A-10 (1972), 5-8.

2. A. Erdélyi et al., Tables of integral transforms, vol. 2, McGraw-Hill, New York, 1954.

3. C. Fox, The $G$ and $H$-functions as symmetrical Fourier kernels, Trans. Amer. Math. Soc. 98 (1961), 395-429.

4. K. C. Gupta and U. C. Jain, The H-function. II, Proc. Nat. Acad. Sci. India A 36 (1966), 594-602. 
5. K. C. Gupta, Ph.D. Thesis, Rajasthan Univ., 1966.

6. K. S. Miller, The Weyl fractional calculus, Fractional Calculus and its Applications, Lecture Notes in Math., vol. 457, Springer-Verlag, New York, 1975, pp. 80-89.

7. T. J. Osler, Taylor's series generalized for fractional derivatives and applications, SIAM J. Math. Anal. 2 (1971), 37-48.

Departmant of Mathimatics, S.K.N. Agriculture Collbge, Jobner-303329, India

Department of MAthematics, M.R. Engineering College, JAIPUR-302004, Indi 